
spin star system

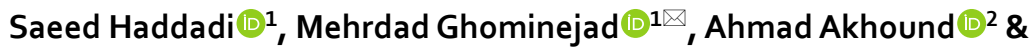 \\ Mohammad Reza Pourkarimi ${ }^{3}{ }^{3}$
}

The uncertainty principle is known as a foundational element of quantum theory, providing a striking lower bound to quantify our prediction for the measured result of two incompatible observables. In this work, we study the thermal evolution of the entropic uncertainty bound in the presence of quantum memory for an inhomogeneous four-qubit spin-star system that is in the thermal regime. Intriguingly, our results show that the entropic uncertainty bound can be controlled and suppressed by adjusting the inhomogeneity parameter of the system.

The uncertainty principle is undoubtedly one of the most fundamental aspects of quantum mechanics introduced by Heisenberg ${ }^{1}$. The first uncertainty relation for the position $\hat{x}$ and the momentum $\hat{p}$ was formulated by Kennard as $\Delta \hat{x} \Delta \hat{p} \geq \hbar / 2^{2}$. Literally, Heisenberg's uncertainty principle states that two arbitrary incompatible observables $Q$ and $R$ cannot be measured accurately at the same time. Later, Robertson ${ }^{3}$ proposed an inequality as $\Delta Q \Delta R \geq|\langle[Q, R]\rangle| / 2$ where $\Delta \mathscr{X}=\sqrt{\left\langle\mathscr{X}^{2}\right\rangle-\langle\mathscr{X}\rangle^{2}}$ is the standard deviation with $\mathscr{X} \in\{Q, R\},\langle\mathscr{X}\rangle$ is the mean value of operator $\mathscr{X}$, and $[Q, R]=Q R-R Q$ is the commutator. In the recent decade, the outstanding achievement accomplished by many authors is a connection between the uncertainty relation and the information theory that is well known as the entropic uncertainty relation (EUR). Deutsch ${ }^{4}, \mathrm{Kraus}^{5}$, Maassen and Uffink ${ }^{6}$ are researchers who have been pioneers in this subject. Formally, the EUR is defined as $H(Q)+H(R) \geq-\log _{2} c$ where $H(Q)=-\sum_{i} q_{i} \log _{2} q_{i}$ and $H(R)=-\sum_{j} r_{j} \log _{2} r_{j}$ are the Shannon entropy of the probabilities of observables $Q$ and $R$ measurement results, respectively. The complementarity parameter $c=\max _{i, j}\left\{\left|\left\langle q_{i} \mid r_{j}\right\rangle\right|^{2}\right\}$ is the maximal overlap of $Q$ and $R$ with $\left|q_{i}\right\rangle$ and $\left|r_{j}\right\rangle$ being the eigenstates of $Q$ and $R$, respectively.

Nevertheless, a new kind of the EUR in the presence of quantum memory (EUR-QM) has been presented by Berta et al. ${ }^{7}$ in which two players, Alice and Bob, play an uncertainty game. In this game, Bob prepares a correlated two-particle state $\rho^{A B}$ and he then sends particle $A$ to Alice which is correlated to his memory particle $B$. After that, Alice measures her particle with respect to operator $Q$ or $R$ and announces to Bob the result of her choice. Hence, Bob can guess and minimize his uncertainty based on Alice's measurement result. This new uncertainty relation can be written as

$$
S(Q \mid B)+S(R \mid B) \geq-\log _{2} c+S(A \mid B),
$$

where $S(A \mid B)=S\left(\rho^{A B}\right)-S\left(\rho^{B}\right)$ being the conditional von Neumann entropy of $\rho^{A B}$ with $\rho^{B}=\operatorname{tr}_{A}\left(\rho^{A B}\right)$ and $S(\rho)=-\operatorname{tr}\left(\rho \log _{2} \rho\right)$ is the von Neumann entropy. Also, $S(Q \mid B)=S\left(\rho^{Q B}\right)-S\left(\rho^{B}\right)$ and $S(R \mid B)=S\left(\rho^{R B}\right)-S\left(\rho^{B}\right)$ are the conditional von Neumann entropies of the post-measurement states $\rho^{Q B}=\sum_{i}\left(\left|q_{i}\right\rangle_{A}\left\langle q_{i}\right| \otimes \mathbb{I}_{B}\right) \rho^{A B}\left(\left|q_{i}\right\rangle_{A}\left\langle q_{i}\right| \otimes \mathbb{I}_{B}\right)$ and $\rho^{R B}=\sum_{j}\left(\left|r_{j}\right\rangle_{A}\left\langle r_{j}\right| \otimes \mathbb{I}_{B}\right) \rho^{A B}\left(\left|r_{j}\right\rangle_{A}\left\langle r_{j}\right| \otimes \mathbb{I}_{B}\right)$ after the quantum system $A$ is measured, and $\mathbb{I}_{B}$ being the identity operator.

Till date, the EUR-QM has been the topic of much work ${ }^{8-48}$ and many attempts have been made to tighten this inequality ${ }^{49-60}$. Moreover, many efforts have been made to generalize the various entropic uncertainty relations to more than two measurements ${ }^{61-67}$. Amongst these encouraging efforts, one can refer to the result obtained by Adabi et al. ${ }^{53}$. In 2016, they proposed a tighter bound which has an extra term, comparing to the inequality (1), viz

$$
S(Q \mid B)+S(R \mid B) \geq-\log _{2} c+S(A \mid B)+\max \{0, \kappa\},
$$

with $\kappa=I(A: B)-[I(Q: B)+I(R: B)]$ where $I(A: B)=S\left(\rho^{A}\right)+S\left(\rho^{B}\right)-S\left(\rho^{A B}\right)$ is mutual information, $I(\mathscr{X}: B)=S\left(\rho^{B}\right)-\sum_{x} p_{x} S\left(\rho_{x}^{B}\right)$ is Holevo quantity, $\rho_{x}^{B}=\operatorname{tr}_{A}\left(\Pi_{x}^{A} \rho^{A B} \Pi_{x}^{A}\right) / p_{x}$ is the post-measurement state

${ }^{1}$ Faculty of Physics, Semnan University, P.O.Box 35195-363, Semnan, Iran. ${ }^{2}$ Department of Physics, Payame Noor University, P.O.Box 19395-3697, Tehran, Iran. ${ }^{3}$ Department of Physics, Salman Farsi University of Kazerun, Kazerun, Iran. ${ }^{凶}$ email: mghominejad@semnan.ac.ir 


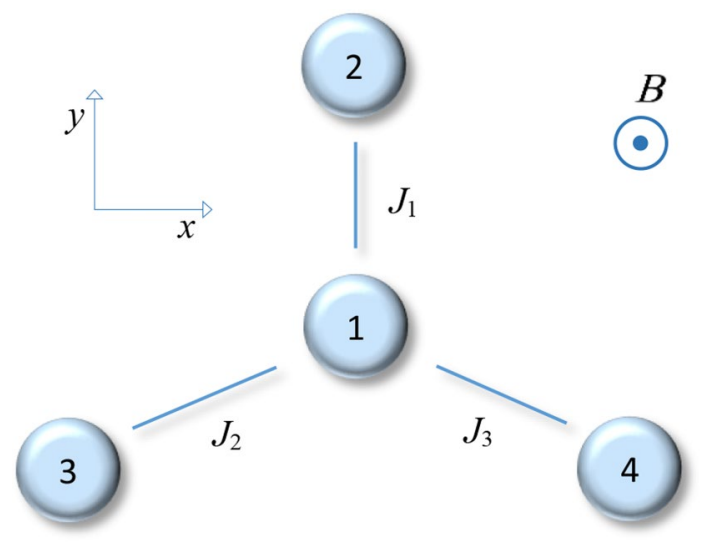

Figure 1. A schematic diagram of a four-qubit spin-star system. The qubits labelled 2-4 are coupled to the central one with $J_{1}, J_{2}$, and $J_{3}$ which are the coupling constants, respectively. The external magnetic field $\boldsymbol{B}$ is perpendicular to the plane where the four qubits lie.

of Bob after measuring of $\mathscr{X}$ by Alice, and $p_{x}=\operatorname{tr}_{A B}\left(\Pi_{x}^{A} \rho^{A B} \Pi_{x}^{A}\right)$ is the probability of $x$ th outcome. Note that the tightness of the uncertainty relation means that the difference between the uncertainty and its bound is the smallest value. In this work, we consider the right-hand side of the inequality (2) as an entropic uncertainty bound, i.e.,

$$
U_{b}\left(\rho^{A B}\right) \equiv-\log _{2} c+S(A \mid B)+\max \{0, \kappa\} .
$$

In recent decades, many researchers have been interested in studying spin systems because of their application in quantum information ${ }^{68-71}$. The spin chain is one of the most popular spin systems in which spins interact with their neighbors, well known as the Heisenberg model, which has been sufficiently reviewed by many authors so far $^{72-84}$. Motivated by this, we study another kind of spin system known as a spin-star system for which spins cannot interact with each other directly, and a central spin is responsible for interacting with the other spins. Historically, the study of the spin-star system started in 2004 when Hutton and Bose ${ }^{85}$ investigated the interesting properties of a physical system that a central spin interacts with the outer ones. In literature, lots of researches have been devoted to investigating quantum correlations in the spin-star system ${ }^{86-96}$, e.g., Anzà et al. ${ }^{93}$ investigated tripartite thermal correlations in an inhomogeneous spin-star system using concurrence and tripartite negativity criteria. The authors examined the dependence of such quantum correlations on the homogeneity and inhomogeneity of the interactions, and they found some interesting differences between the tripartite negativity and concurrence. Moreover, Militello and Messina ${ }^{94}$ examined the tripartite thermal entanglement in an inhomogeneous spin-star network with three external spins. From a practical point of view, the spin-star system can be realized in many solid state systems, such as the nitrogen-vacancy centre in diamond ${ }^{97,98}$ and the semiconductor quantum dot ${ }^{99-101}$ (see recent paper ${ }^{102}$ and references therein for a detailed study on the spinstar system). However, to the best of our knowledge, nobody has previously examined the entropic uncertainty for a spin-star system up to now. Because of the lack of such exploring, we are then motivated to investigate the entropic uncertainty bound in a four-qubit spin-star system and finding a suitable parameter to control the entropic uncertainty of the system. Hence, this paper is prepared as follows. In "Physical scenario", we consider a four-qubit spin-star system where three outer qubits are coupled to the central one with different strengths, and in "Results and discussion", thermal entropic uncertainty bound in different situations is analyzed. Finally, we discuss our results and present some conclusive remarks subsequently.

\section{Physical scenario}

This section considers a four-qubit spin-star system for which the schematic diagram of such a quantum system is sketched in Fig. 1. The total Hamiltonian of the mentioned system is given by ( $\operatorname{setting} \hbar=1$ )

$$
\mathbf{H}=\sum_{i=1}^{3} J_{i}\left(\sigma_{1}^{+} \sigma_{i+1}^{-}+\sigma_{1}^{-} \sigma_{i+1}^{+}\right)+\boldsymbol{B} \sum_{i=1}^{4} \sigma_{i}^{z},
$$

where $J_{1}, J_{2}$, and $J_{3}$ are the coupling constants of the central qubit with the outer qubits 2,3 , and 4 , respectively. Besides, $\boldsymbol{B}$ indicates the magnitude of the external magnetic field, $\sigma_{i}^{z}(i=1,2,3,4)$ is the Pauli operator in the $z$-direction for $i$ th qubit, and $\sigma_{i}^{+}$as well as $\sigma_{i}^{-}$are the ladder operators.

Let us assume that a typical system reaches thermodynamical equilibrium. So, its density operator can be described by the thermal state as

$$
\rho_{T}=\frac{1}{\mathscr{Z}} \exp \left(-\mathbf{H} / \kappa_{B} T\right)=\frac{1}{\mathscr{Z}} \sum_{i} \exp \left(-E_{i} / \kappa_{B} T\right)\left|\psi_{i}\right\rangle\left\langle\psi_{i}\right|,
$$


where $\mathscr{Z}=\operatorname{tr}\left[\exp \left(-\mathbf{H} / \kappa_{B} T\right)\right]$ is the system partition function with $\kappa_{B}$ as the Boltzmann constant, considered $\kappa_{B}=1$ by us, as it is used in the natural unit system. Moreover, $E_{i}$ and $\left|\psi_{i}\right\rangle$ are the eigenvalues and the eigenstates of the total Hamiltonian, respectively.

Now, we consider an inhomogeneous Hamiltonian for which $J_{1}=J_{3}=J$ and $J_{2}=x J$, where $x$ is a suitable dimensionless inhomogeneity parameter. It is clear that the homogeneous Hamiltonian is obtained for $x=1$. Having known the Hamiltonian (4) and assuming inhomogeneity, the results of the diagonalization of $\mathbf{H}$ are reported in "Methods". Then, by using Eq. (5) and through taking partial traces over the outer two qubits for a four-qubit thermal state, the reduced density matrices in the standard basis $\{|00\rangle,|01\rangle,|10\rangle,|11\rangle\}$ with $A=1$ and $B=2,3,4$ are given in "Methods". It is quite obvious that due to the inhomogeneity, $\rho_{T}^{12}=\rho_{T}^{14} \neq \rho_{T}^{13}$, so let's call $\rho_{T, 1}=\rho_{T}^{12}=\rho_{T}^{14}$ as case 1 and $\rho_{T, 2}=\rho_{T}^{13}$ as case 2 , henceforth.

\section{Results and discussion}

Here, we would like to investigate the thermal evolution of the entropic uncertainty bound by considering two thermal quantum states $\left(\rho_{T, 1}\right.$ and $\left.\rho_{T, 2}\right)$. In each of the related subsections, we will explicitly present and discuss the analytical results.

Model with inhomogeneity of case 1. Based upon the results of the previous section, we can now analyze the thermal evolution of the entropic uncertainty bound for the quantum state $\rho_{T, 1}$ in this section. In this scenario, Bob prepares a correlated two-qubit state $\rho_{T, 1}$ and then he sends one qubit to Alice and keeps the other one as a quantum memory in his hand. Herein, without loss of the generality, we consider the situation where Alice measures one of the two observables $Q=\sigma^{x}$ or $R=\sigma^{z}$ where $\sigma^{x}$ and $\sigma^{z}$ are the Pauli operators and then, we will obtain the complementarity parameter as $c=1 / 2$. The thermal reduced density matrix $\rho_{T}^{12}=\rho_{T}^{14}=\operatorname{tr}_{34}\left(\rho_{T}\right)=\operatorname{tr}_{23}\left(\rho_{T}\right)$ is obtained as

$$
\rho_{T, 1}=\rho_{T}^{12}=\rho_{T}^{14}=\left(\begin{array}{cccc}
v & 0 & 0 & 0 \\
0 & u^{+} & w & 0 \\
0 & w & u^{-} & 0 \\
0 & 0 & 0 & q
\end{array}\right)
$$

Note that, the density matrix components are reported in "Methods". Obviously now, by substituting the elements of the thermal reduced density matrix (6) into Eq. (3), one can obtain the analytical expression of the entropic uncertainty bound as what follows

$$
U_{b}\left(\rho_{T, 1}\right)=\Delta_{T, 1}+\max \left\{0, \Theta_{T, 1}\right\},
$$

where

$$
\Delta_{T, 1}=1-H_{2}\left(v+u^{-}\right)-\sum_{i} \tau_{i} \log _{2} \tau_{i}
$$

and

$$
\Theta_{T, 1}=-H_{2}\left(v+u^{-}\right)+H_{2}\left(\frac{1+k}{2}\right)-\sum_{i} \rho_{T, 1(i i)} \log _{2} \rho_{T, 1(i i)}+\sum_{i} \tau_{i} \log _{2} \tau_{i},
$$

where $\tau_{i}$ 's are the eigenvalues of $\rho_{T, 1}$ and $k=\sqrt{4|w|^{2}+\left[1-2\left(q+u^{+}\right)\right]^{2}}$. Above, $S\left(\rho_{T, 1}^{B}\right)=H_{2}\left(v+u^{-}\right)$is the von Neumann entropy of quantum memory and $H_{2}(\varepsilon)=-\epsilon \log _{2} \varepsilon-(1-\varepsilon) \log _{2}(1-\varepsilon)$ for any $\varepsilon \in[0,1]$ is the binary Shannon entropy function.

Up to now, we have analytically derived the entropic uncertainty bound for a quantum state $\rho_{T, 1}$. However, our results show that to possess an exact solution for that, depends on the different parameters of the system under study. Hence, let us analyze the entropic uncertainty bound as functions of the temperature and the system parameters.

Figure 2 shows the thermal evolution of the entropic uncertainty bound (7) as a function of the inhomogeneity parameter $(x)$ for specific values of the temperature $(T)$, external magnetic field $(\boldsymbol{B})$, and the coupling constant $(J)$ between the central spin and the peripheral ones. Following, Fig. 2a shows that the entropic uncertainty bound of $\rho_{T, 1}$ grows with increasing the temperature, and hence, Bob's uncertainty about Alice's measurement outcome increases. Of course, this is not surprising, as the increasing temperature can reduce quantum correlations, leading to an increase in measurement uncertainty of incompatible observables. It is quite clear that the physical reason for increasing the uncertainty with respect to temperature, is the high degree of mixedness. Compared to temperature, here the role of the external magnetic field is quite constructive to predict the results of Alice's measurement by Bob, as seen from Fig. 2b. The role of the coupling constant, as shown in Fig. 2c, is quite complicated. This means that the entropic uncertainty bound for $x<1$ increases with increasing the coupling constant, but for $x>1$ the event is different. In fact, when $x$ is much greater than $1, J_{2}=x J$ is much greater than $J_{1}=J_{3}=J$, and so, roughly speaking, qubits 2 and 4 can be considered almost decoupled, and then separable. For this reason, the increase in the inhomogeneity parameter notably leads to reducing Bob's ability to predict Alice's measurement outcome, as can be seen from all plots. It is worth noting that for $x=1$, the system under study is a homogeneous model.

For more clarification, we have drawn contour diagrams of the entropic uncertainty bound as functions of the inhomogeneity parameter $x$ and different values of the temperature, external magnetic field, and the coupling constant in Fig. 3. As depicted in Fig. 3a, the entropic uncertainty bound increases dramatically with the growth 
(a)

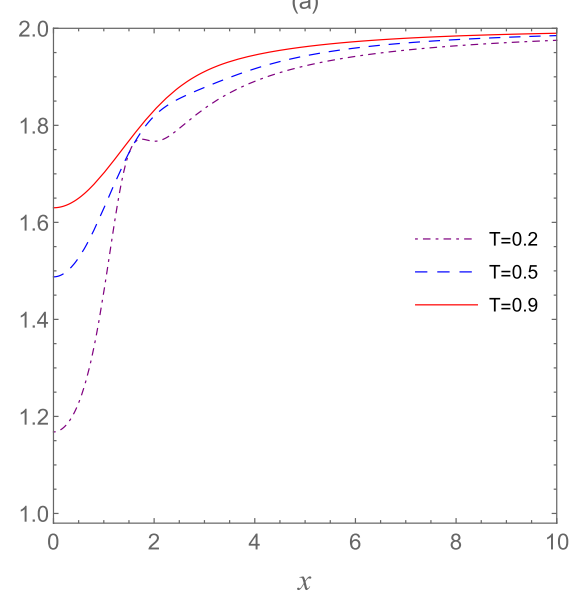

(b)

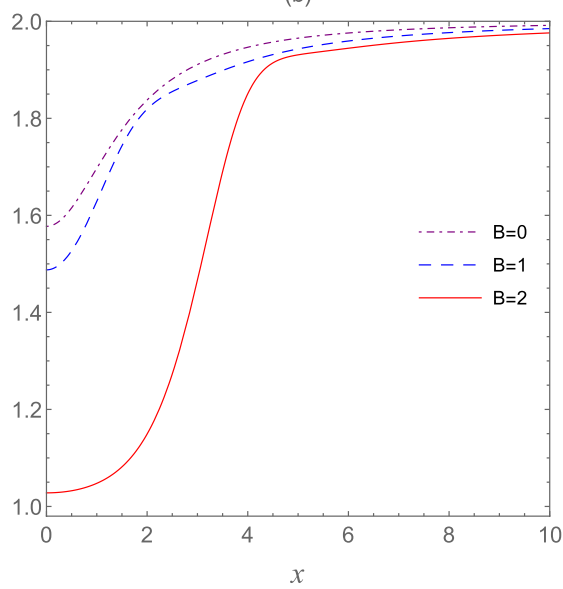

(c)

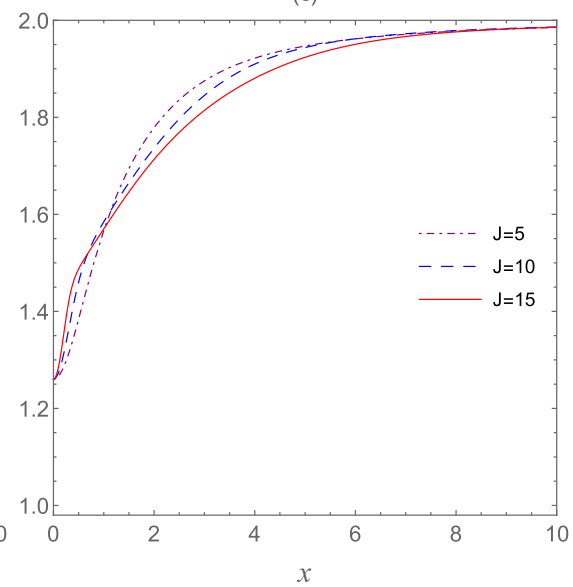

Figure 2. The entropic uncertainty bound $U_{b}\left(\rho_{T, 1}\right)$ as a function of the inhomogeneity parameter $x$ for fixed values of the temperature $T$, external magnetic field $\boldsymbol{B}$, and the coupling constant $J$. Graph (a) $\boldsymbol{B}=1 ; J=1$, graph $(\mathbf{b}) T=0.5 ; J=1$, and graph $(\mathbf{c}) T=0.5 ; \boldsymbol{B}=1$.

(a)

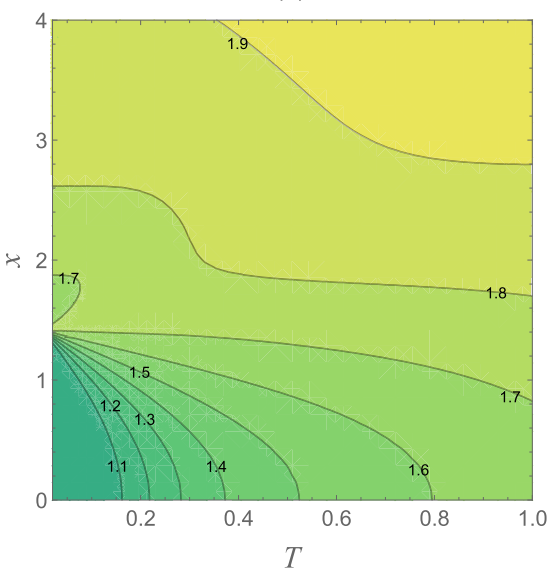

(b)

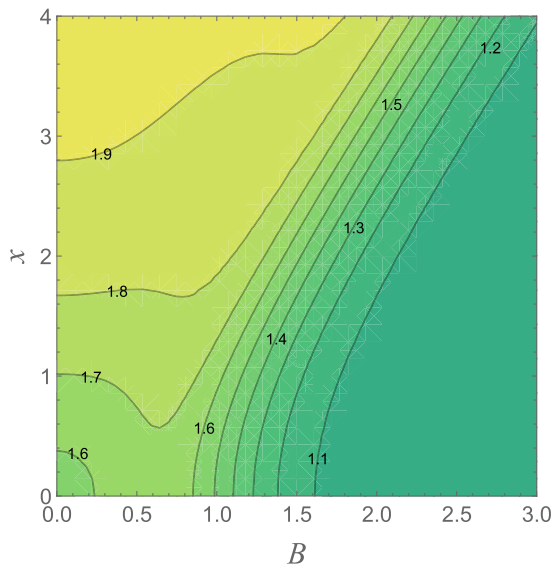

(c)

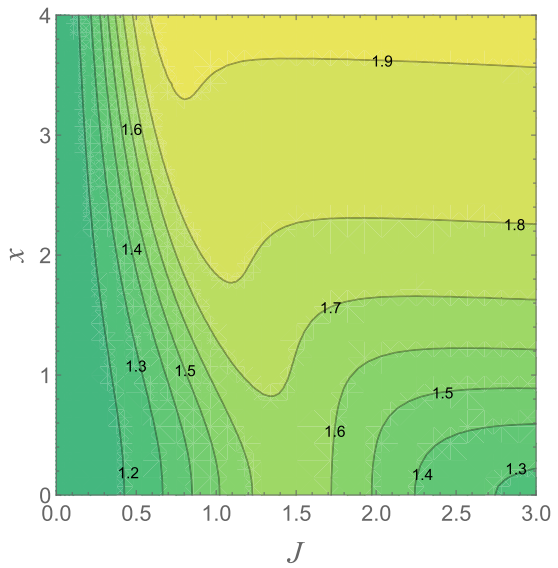

Figure 3. The entropic uncertainty bound $U_{b}\left(\rho_{T, 1}\right)$ as a function of the inhomogeneity parameter $x$ and different values of the temperature $T$, external magnetic field $\boldsymbol{B}$, and the coupling constant $J$. Graph (a) $\boldsymbol{B}=1$; $J=1$, graph $(\mathbf{b}) T=0.5 ; J=1$, and graph $(\mathbf{c}) T=0.5 ; \boldsymbol{B}=1$.

in the inhomogeneity parameter $x$ and temperature value, with $\boldsymbol{B}=1$ and $J=1$, which is obviously in accordance with what mentioned above. In Fig. $3 \mathrm{~b}$, the entropic uncertainty bound as functions of $x$ and $\boldsymbol{B}$ has been plotted. It directly shows that the smaller $x$ and the greater $\boldsymbol{B}$ can both induce Bob's uncertainty about Alice's measurement result to become less, which is desired in practical quantum information processing. Let us now turn to focus on the influence of the inhomogeneity parameter $x$ and the strength of the coupling parameter on the entropic uncertainty bound, as shown in Fig. 3c. At a fixed temperature and $\boldsymbol{B}=1$, in the $0<J<1 / 2$ area, the entropic uncertainty bound is somewhat insensitive to the inhomogeneity parameter variations. However, in the $1 / 2 \leq J \leq 3$ area, the inhomogeneity parameter appears to be a dominant factor.

Model with inhomogeneity of case 2. According to the previous analysis, we can obtain the analytical expression of the entropic uncertainty bound for the quantum state $\rho_{T, 2}$. The thermal reduced density matrix $\rho_{T}^{13}=\operatorname{tr}_{24}\left(\rho_{T}\right)$ is taken as follows

$$
\rho_{T, 2}=\rho_{T}^{13}=\left(\begin{array}{cccc}
\vartheta & 0 & 0 & 0 \\
0 & \mu^{+} & v & 0 \\
0 & \nu & \mu^{-} & 0 \\
0 & 0 & 0 & \xi
\end{array}\right),
$$

where the non-zero elements are given again in "Methods". By substituting the elements of the thermal reduced density matrix (10) into Eq. (3), it is again easy to see that 
(a)

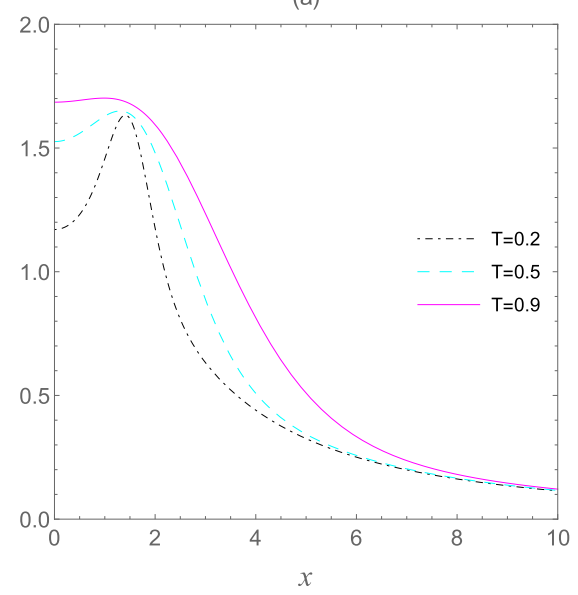

(b)

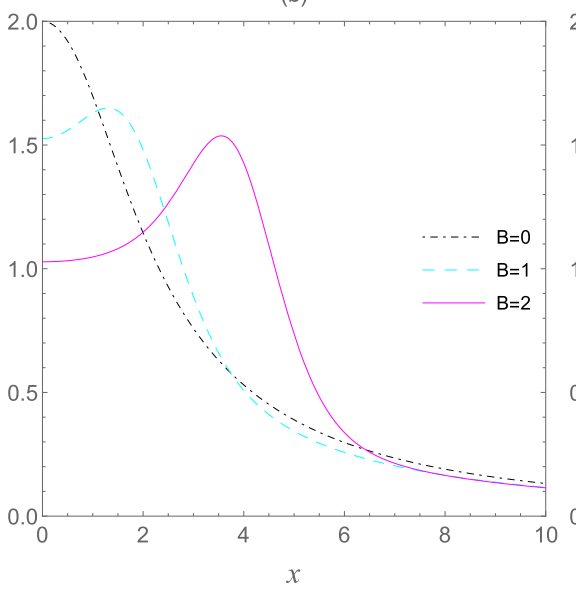

(c)

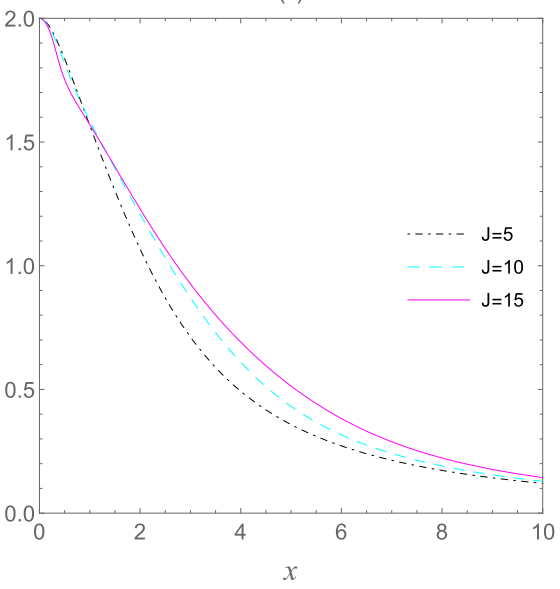

Figure 4. The entropic uncertainty bound $U_{b}\left(\rho_{T, 2}\right)$ as a function of the inhomogeneity parameter $x$ for fixed values of the temperature $T$, external magnetic field $\boldsymbol{B}$, and the coupling constant $J$. Graph $(\mathbf{a}) \boldsymbol{B}=1 ; J=1$, graph $(\mathbf{b}) T=0.5 ; J=1$, and graph $(\mathbf{c}) T=0.5 ; \boldsymbol{B}=1$.

$$
U_{b}\left(\rho_{T, 2}\right)=\Delta_{T, 2}+\max \left\{0, \Theta_{T, 2}\right\},
$$

where

$$
\Delta_{T, 2}=1-H_{2}\left(\vartheta+\mu^{-}\right)-\sum_{i} \chi_{i} \log _{2} \chi_{i}
$$

and

$$
\Theta_{T, 2}=-H_{2}\left(\vartheta+\mu^{-}\right)+H_{2}\left(\frac{1+\varsigma}{2}\right)-\sum_{i} \rho_{T, 2(i i)} \log _{2} \rho_{T, 2(i i)}+\sum_{i} \chi_{i} \log _{2} \chi_{i},
$$

where $\chi_{i}^{\prime}$ s are the eigenvalues of $\rho_{T, 2}$ and $\varsigma=\sqrt{4|\nu|^{2}+\left[1-2\left(\xi+\mu^{+}\right)\right]^{2}}$. Here, $S\left(\rho_{T, 2}^{B}\right)=H_{2}\left(\vartheta+\mu^{-}\right)$denotes the von Neumann entropy of quantum memory.

In Fig. 4, we first examine the effect of temperature on the entropic uncertainty of interest and draw the thermal evolution of the entropic uncertainty bound $U_{b}\left(\rho_{T, 2}\right)$ as functions of inhomogeneity parameter $x$ and fixed values of $T$ in Fig. $4 \mathrm{a}$. As the previous case, one can observe that the entropic uncertainty bound increases by growing the temperature. On the other hand, in analyzing the effects of the inhomogeneity parameter $x$ on the entropic uncertainty, we observe that the entropic uncertainty grows when the value of $x$ enhances at lower temperatures, and after passing a peak, it becomes smaller and quickly achieves a steady value for the larger amounts of $x$. As plotted in Fig. $4 \mathrm{~b}$, for $\boldsymbol{B}=1$ and $\boldsymbol{B}=2$, it is obvious that the entropic uncertainty bound is firstly increased and then reduces as $x$ raises. However, in the absence of an external magnetic field $(\boldsymbol{B}=0)$, the uncertainty bound monotonously decreases when $x$ grows. In order to further probe the relationship between the uncertainty bound and the inhomogeneity $x$ as well as the coupling coefficient $J$, we have drawn the uncertainty bound as a function of $x$ for three values of $J$, as displayed in Fig. $4 \mathrm{c}$. At fixed temperature and $\boldsymbol{B}=1$, in the $0<x<1$ area, while $x$ is much smaller than $1, J_{2}=x J$ is much smaller than $J_{1}=J_{3}=J$, and hence the qubit 3 can be considered almost decoupled, and then the uncertainty bound is maximal for $x=0$. Nevertheless, for $x$ greater than 1, the coupling between the central qubit and the qubit 3 becomes more robust than the other two, and hence the measurement uncertainty is significantly suppressed.

To further study the relationship between the uncertainty bound and the inhomogeneity $x$ as well as the other parameters of the thermal system, we have drawn the contour diagrams of the uncertainty bound as functions of $T, \boldsymbol{B}$, and $J$ in plots (a) to (c) respectively, as shown in Fig. 5. One can readily observe that the uncertainty bound decreases with the reducing $T$, but the role of the external magnetic field $\boldsymbol{B}$ and the coupling coefficient $J$ seem to be somewhat complex. Hence, we can see that these achieved results are well in agreement with the report we made earlier in Fig. 4. In short, regardless of the temperature values and the values of $\boldsymbol{B}$ and $J$, the greater inhomogeneity parameter $x$ can induce a smaller uncertainty bound, and vice versa. This result apparently implies that the maximum symmetry in the four-qubit spin-star system does not correspond to the minimum uncertainties between all its parts, i.e., the uncertainties between qubits $1-2,1-3$, and 1-4.

Comparison of case 1 with case 2. After reviewing the analytical and numerical results, let us now compare the thermal evolution of the entropic uncertainty bound in two cases. From the outlook of quantitative description of the entropic uncertainty bound, we need to state that in the thermal state $\rho_{T, 1}$, regardless of the temperature, $\boldsymbol{B}$, and $J$, the uncertainty bound increases and reach a maximum fixed value (saturated value) when the inhomogeneity $x$ grows $\left(U_{b}\left(\rho_{T, 1}\right) \simeq 2\right)$. Therefore, in this case, Bob's information about the result of Alice's measurement is reduced rapidly. However, in the case of the thermal state $\rho_{T, 2}$, the uncertainty bound comes 
(a)

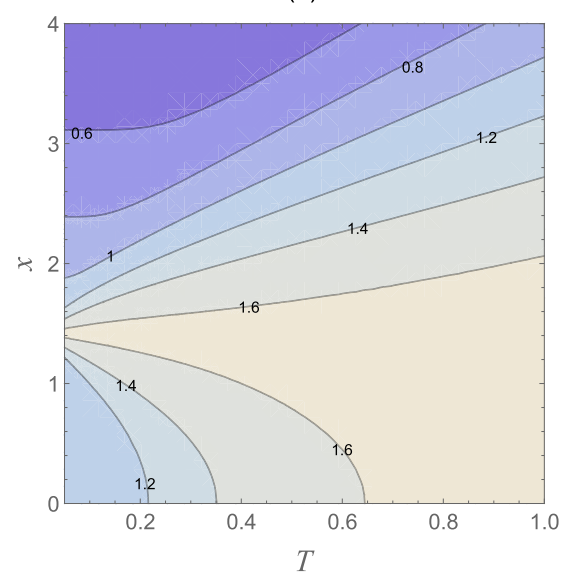

(b)

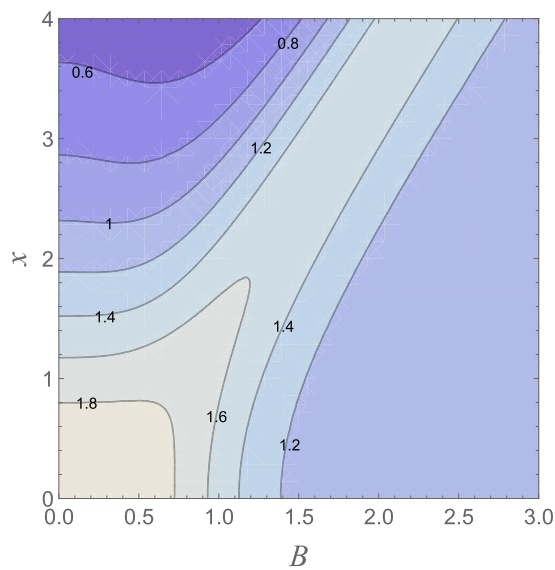

(c)

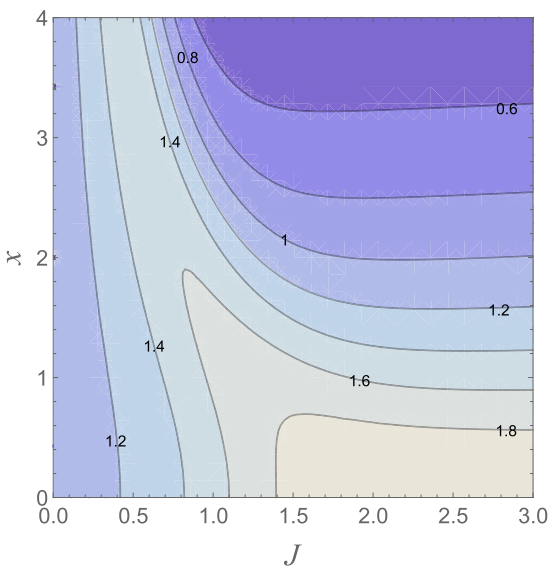

Figure 5. The entropic uncertainty bound $U_{b}\left(\rho_{T, 2}\right)$ as a function of the inhomogeneity parameter $x$ and different values of the temperature $T$, external magnetic field $\boldsymbol{B}$, and the coupling constant $J$. Graph (a) $\boldsymbol{B}=1$; $J=1$, graph $(\mathbf{b}) T=0.5 ; J=1$, and graph $(\mathbf{c}) T=0.5 ; \boldsymbol{B}=1$.

near to zero when the inhomogeneity $x$ increases. The physical reason is that as long as the inhomogeneity $x$ grows, the coupling between the central qubit and the qubit 3 becomes stronger than the other two, and therefore $U_{b}\left(\rho_{T, 2}\right) \rightarrow 0$ while $U_{b}\left(\rho_{T, 1}\right) \rightarrow 2$. It is worth noting that in this situation $\left(\rho_{T, 2}\right)$, Bob's uncertainty about Alice's measurement outcome remarkably decreases. Hence, we find that the uncertainty bound can be controlled by manipulating the inhomogeneity parameter and then Bob can accurately guess Alice's measurement outcome.

Physical interpretation and possible applications. In this work, we have examined the features of thermal entropic uncertainties in the presence of inhomogeneity since in real conditions the construction of a system with ideally homogeneous interactions could be much challenging. Accordingly, we have shown that the measurement uncertainty of incompatible observables is controllable by regulating the inhomogeneity parameter. This implies the idea that the thermal uncertainties mediated by the central spin can be remarkably controlled by a certain absence of homogeneity that could describe a more realistic situation, even if the degree of inhomogeneity is high. Hence, our result may be advantageous for practical quantum information processing, and it is physically realizable in different arrays of interacting particles designed for quantum computing ${ }^{103}$. Notably, controlling the entropic uncertainty has some versatile applications in practical quantum tasks including, quantum cryptography ${ }^{104}$, entanglement witness ${ }^{105}$, quantum key distribution ${ }^{106}$, quantum metrology ${ }^{107}$, quantum teleportation ${ }^{108}$, quantum steering ${ }^{109}$, and so on ${ }^{110}$. Specifically, measurement of uncertainty in a spinstar system can be experimentally realized by molecular nanomagnets, NMR molecules, superconducting spins, and coupled microcavities ${ }^{111}$.

\section{Conclusions and remarks}

To conclude, we have studied the entropic uncertainty bound in a spin-star system with three peripheral qubits, all affected by an external magnetic field. The interaction with the central qubit is responsible for establishing tripartite correlations between the outer ones, and such correlations remain even if the system is at thermal equilibrium. We have considered an inhomogeneous model, where one of the peripheral qubits is coupled to the central one with a different strength. In the following, we analytically derived the entropic uncertainty bound for the quantum states $\rho_{T, 1}$ and $\rho_{T, 2}$ and then we investigated the thermal evolution of the entropic uncertainty bound in measuring two incompatible observables under the effect of the temperature $T$, the magnetic field $\boldsymbol{B}$, the coupling coefficient strength $J$, and the inhomogeneity parameter $x$. A remarkable point is, we found that the entropic uncertainty bound can be improved by changing the inhomogeneity as an efficient control parameter. Therefore, we think that a higher degree of symmetry in the system does not guarantee fewer uncertainties between all its parts. This issue could be highly instructive and available to quantum precision measurement by adjusting proper measurement parameters and conditions.

\section{Methods}

Eigenvalues and the eigenstates of the Hamiltonian. In this section, we give the eigenvalues $E_{i}$ and the eigenstates $\left|\psi_{i}\right\rangle$ of the inhomogeneous Hamiltonian as functions of $x>0, J>0$, and $\boldsymbol{B}$. The homogeneous model is achieved for $x=1$. The eigenvalues of the Hamiltonian are

$$
\begin{aligned}
& E_{1}=2 \boldsymbol{B}, \quad E_{2}=-2 \boldsymbol{B}, \quad E_{3}^{ \pm}= \pm J x, \quad E_{4}^{ \pm}= \pm \frac{J}{2}\left(x+\sqrt{x^{2}+8}\right), \quad E_{5}^{ \pm}= \pm \frac{J}{2}\left(x-\sqrt{x^{2}+8}\right), \\
& E_{6}^{ \pm}=2 \boldsymbol{B} \pm J \sqrt{x^{2}+2}, \quad E_{7}^{ \pm}=-2 \boldsymbol{B} \pm J \sqrt{x^{2}+2}, \quad E_{8}=-4 \boldsymbol{B}, \quad E_{9}=4 \boldsymbol{B},
\end{aligned}
$$

where the eigenvalues $E_{1}$ and $E_{2}$ are twofold degenerate eigenvalues. The relevant eigenstates are 


$$
\begin{gathered}
\left|\psi_{1}^{a}\right\rangle=\frac{1}{M_{1}}\left[-\frac{1}{x}|0001\rangle-|0010\rangle+\left(\frac{1}{x}+x\right)|0100\rangle\right], \\
\left|\psi_{1}^{b}\right\rangle=\frac{1}{\sqrt{x^{2}+1}}(-x|0001\rangle+|0010\rangle), \\
\left|\psi_{2}^{a}\right\rangle=\frac{1}{M_{1}}\left[-\frac{1}{x}|1011\rangle-|1101\rangle+\left(\frac{1}{x}+x\right)|1110\rangle\right], \\
\left|\psi_{2}^{b}\right\rangle=\frac{1}{\sqrt{x^{2}+1}}(-x|1011\rangle+|1101\rangle), \\
\left|\psi_{3}^{ \pm}\right\rangle=\frac{1}{M_{2}}\left[(|1001\rangle \pm|0011\rangle)+(|1100\rangle \pm|0110\rangle)+\frac{\sqrt{x^{2}+8}-x}{2}(|1010\rangle \pm|0101\rangle)\right], \\
\left|\psi_{5}^{ \pm}\right\rangle=\frac{1}{M_{2}}\left[(|1001\rangle \pm|0011\rangle)+(|1100\rangle \pm|0110\rangle)-\frac{\sqrt{x^{2}+8}+x}{2}(|1010\rangle \pm|0101\rangle)\right], \\
\left|\psi_{6}^{ \pm}\right\rangle=\frac{1}{M_{3}}\left[\sqrt{x^{2}+2}|1000\rangle \pm(|0001\rangle+x|0010\rangle+|0100\rangle)\right], \\
\left|\psi_{7}^{ \pm}\right\rangle=\frac{1}{M_{3}}\left[(|1011\rangle+x|1101\rangle+|1110\rangle) \pm \sqrt{x^{2}+2}|0111\rangle\right], \\
\left|\psi_{8}\right\rangle=|0000\rangle,|1111\rangle, \\
|(|001\rangle \pm|0011\rangle)],
\end{gathered}
$$

with

$$
M_{1}^{2}=\frac{2}{x^{2}}+3+x^{2}, \quad M_{2}^{2}=8+x\left(x-\sqrt{x^{2}+8}\right), \quad M_{3}^{2}=2\left(2+x^{2}\right) .
$$

Matrix elements of thermal reduced density matrices. Here, we give the matrix elements of the thermal reduced density matrices $\left(\rho_{T}^{12}=\rho_{T}^{14} \neq \rho_{T}^{13}\right)$ for the inhomogeneous Hamiltonian as functions of $x, J>0$, and $\boldsymbol{B}$. The matrix elements of thermal reduced density matrix $\rho_{T, 1} \mathrm{read}$

$$
\begin{aligned}
& v= \frac{1}{2 \mathscr{Z}}\left(\cosh [4 \gamma]+\cosh [x \delta]+2 \eta^{-1} e^{-2 \gamma}(\eta+1+(\eta-1) \cosh [\delta \sqrt{\eta}])\right. \\
&\left.+\cosh [x \delta / 2] \cosh [\delta \sqrt{\theta} / 2]-2 \sinh [4 \gamma]+x \theta^{-\frac{1}{2}} \sinh [x \delta / 2] \sinh [\delta \sqrt{\theta} / 2]\right) \\
& u^{ \pm}=\frac{1}{4 \mathscr{Z} \eta \theta}\left(4 \cosh [2 \gamma]\left(8+9 x^{2}+x^{4}+(\eta+1) \theta \cosh [\delta \sqrt{\eta}]\right)+2 \eta \theta(\cosh [x \delta]+3 \cosh [x \delta / 2] \cosh [\delta \sqrt{\theta} / 2])\right. \\
&+x^{3} \sqrt{\theta}\left(\cosh \left[\delta \alpha_{-} / 2\right]-\cosh \left[\delta \alpha_{+} / 2\right]\right)+2 x \sqrt{\theta}\left(\cosh \left[\delta \alpha_{-} / 2\right]-\cosh \left[\delta \alpha_{+} / 2\right]\right) \\
&\left. \pm 8\left(8+9 x^{2}+x^{4}\right) \sinh [2 \gamma] \sinh ^{2}[\eta \delta / 2]\right) \\
& q= \\
&+\frac{1}{4 \mathscr{Z} \eta \theta}(4 \eta \theta(\sinh [4 \gamma]+\cosh [4 \gamma])+2 \eta \theta(\cosh [x \delta]+\cosh [x \delta / 2] \cosh [\delta \sqrt{\theta} / 2]) \\
&+x^{3} \sqrt{\theta}\left(\cosh \left[\delta \alpha_{+} / 2\right]-\cosh \left[\delta \alpha_{-} / 2\right]\right)+2 x \sqrt{\theta}\left(\cosh \left[\delta \alpha_{+} / 2\right]-\cosh \left[\delta \alpha_{-} / 2\right]\right) \\
&+4 \theta(\sinh [2 \gamma]+\cosh [2 \gamma])(\eta+1+(\eta-1) \cosh [\delta \sqrt{\eta}]))
\end{aligned}
$$




$$
w=\frac{1}{\mathscr{Z}}\left(-2 \eta^{-\frac{1}{2}} \cosh [2 \gamma] \sinh [\delta \sqrt{\eta}]-4 \theta^{-\frac{1}{2}} \cosh [x \delta / 2] \sinh [\delta \sqrt{\theta} / 2]\right),
$$

besides, the matrix elements of thermal reduced density matrix $\rho_{T, 2}$ read

$$
\begin{aligned}
& \vartheta=\frac{1}{\mathscr{Z}}\left(e^{-4 \gamma}+\eta^{-1}\left(e^{-2 \gamma+\delta \sqrt{\eta}}+e^{-2 \gamma-\delta \sqrt{\eta}}+2(\eta-1) e^{-2 \gamma}\right)\right. \\
& \left.+\frac{1}{2}\left[\cosh \left[\delta \alpha_{+} / 2\right]\left(1-x \theta^{-\frac{1}{2}}\right)+\cosh \left[\delta \alpha_{-} / 2\right]\left(1+x \theta^{-\frac{1}{2}}\right)\right]\right), \\
& \mu^{ \pm}=\frac{1}{\mathscr{Z}}\left(\cosh [x \delta]+2 \eta^{-1} \cosh [2 \gamma](1+(\eta-1) \cosh [\delta \sqrt{\eta}])\right. \\
& +\cosh [x \delta / 2] \cosh [\delta \sqrt{\theta} / 2]+x \theta^{-\frac{1}{2}} \sinh [x \delta / 2] \sinh [\delta \sqrt{\theta} / 2] \\
& \left. \pm 4 \eta^{-1} \sinh [2 \gamma] \sinh ^{2}[\delta \sqrt{\eta} / 2]\right) \\
& \xi=\frac{1}{2 \mathscr{Z} \eta \theta}(2 \eta \theta(\sinh [4 \gamma]+\cosh [4 \gamma])+2 \eta \theta \cosh [x \delta / 2] \cosh [\delta \sqrt{\theta} / 2] \\
& +x^{3} \sqrt{\theta}\left(\cosh \left[\delta \alpha_{-} / 2\right]-\cosh \left[\delta \alpha_{+} / 2\right]\right)+2 x \sqrt{\theta}\left(\cosh \left[\delta \alpha_{-} / 2\right]-\cosh \left[\delta \alpha_{+} / 2\right]\right) \\
& +4 \theta(\sinh [2 \gamma]+\cosh [2 \gamma])(\eta-1+\cosh [\delta \sqrt{\eta}])) \\
& v=\frac{1}{\mathscr{Z}}\left(-\cosh [\delta \sqrt{\theta} / 2] \sinh [x \delta / 2]-\sinh [x \delta]-2 x \eta^{-\frac{1}{2}} \cosh [2 \gamma] \sinh [\delta \sqrt{\eta}]\right. \\
& \left.-x \theta^{-\frac{1}{2}} \cosh [x \delta / 2] \sinh [\delta \sqrt{\theta} / 2]\right) \text {, }
\end{aligned}
$$

with

$$
\mathscr{Z}=2(\cosh [4 \gamma]+\cosh [x \delta]+2 \cosh [2 \gamma](1+\cosh [\delta \sqrt{\eta}])+2 \cosh [x \delta / 2] \cosh [\delta \sqrt{\theta} / 2]),
$$

and

$$
\eta=2+x^{2}, \quad \theta=8+x^{2}, \quad \alpha_{ \pm}=x \pm \sqrt{\theta}, \quad \gamma=B / T, \quad \delta=J / T .
$$

\section{Data availability}

All data generated or analysed during this study are included in this paper.

Received: 26 August 2021; Accepted: 9 November 2021

Published online: 22 November 2021

\section{References}

1. Heisenberg, W. Über den anschaulichen Inhalt der quantentheoretischen Kinematik und Mechanik. Z. Physik 43, 172 (1927).

2. Kennard, E. H. Zur Quantenmechanik einfacher Bewegungstypen. Z. Physik 44, 326 (1927).

3. Robertson, H. P. The uncertainty principle. Phys. Rev. 34, 163 (1929).

4. Deutsch, D. Uncertainty in quantum measurements. Phys. Rev. Lett. 50, 631 (1983).

5. Kraus, K. Complementary observables and uncertainty relations. Phys. Rev. D 35, 3070 (1987).

6. Maassen, H. \& Uffink, J. B. M. Generalized entropic uncertainty relations. Phys. Rev. Lett. 60, 1103 (1988),

7. Berta, M., Christandl, M., Colbeck, R., Renes, J. M. \& Renner, R. The uncertainty principle in the presence of quantum memory. Nat. Phys. 6, 659 (2010).

8. Hu, M. L. \& Fan, H. Quantum-memory-assisted entropic uncertainty principle, teleportation, and entanglement witness in structured reservoirs. Phys. Rev. A 86, 032338 (2012).

9. Hu, M. L. \& Fan, H. Competition between quantum correlations in the quantum-memory-assisted entropic uncertainty relation. Phys. Rev. A 87, 022314 (2013).

10. Hu, M. L. \& Fan, H. Upper bound and shareability of quantum discord based on entropic uncertainty relations. Phys. Rev. A 88, 014105 (2013).

11. Jizba, P., Dunningham, J. A. \& Joo, J. Role of information theoretic uncertainty relations in quantum theory. Ann. Phys. 355, 87 (2015).

12. Jizba, P., Ma, Y., Hayes, A. \& Dunningham, J. A. One-parameter class of uncertainty relations based on entropy power. Phys. Rev. E 93, 060104(R) (2016).

13. Zhang, J., Zhang, Y. \& Yu, C. S. Entropic uncertainty relation and information exclusion relation for multiple measurements in the presence of quantum memory. Sci. Rep. 5, 11701 (2015).

14. Wang, D. et al. Quantum-memory-assisted entropic uncertainty relation in a Heisenberg XYZ chain with an inhomogeneous magnetic field. Laser Phys. Lett. 14, 065203 (2017). 
15. Wang, D., Ming, F., Huang, A. J., Sun, W. Y. \& Ye, L. Entropic uncertainty for spin-1/2 XXX chains in the presence of inhomogeneous magnetic fields and its steering via weak measurement reversals. Laser Phys. Lett. 14, 095204 (2017).

16. Wang, D. et al. Exploration of quantum-memory-assisted entropic uncertainty relations in a noninertial frame. Laser Phys. Lett. 14, 055205 (2017).

17. Chen, P. F. et al. Observation of quantum-memory-assisted entropic uncertainty relation under open systems, and its steering. Laser Phys. Lett. 15, 015206 (2018).

18. Ming, F., Wang, D., Huang, A. J., Sun, W. Y. \& Ye, L. Decoherence effect on quantum-memory-assisted entropic uncertainty relations. Quantum Inf. Process. 17, 9 (2018).

19. Zhang, Y., Fang, M., Kang, G. \& Zhou, Q. Controlling quantum memory-assisted entropic uncertainty in non-Markovian environments. Quantum Inf. Process. 17, 62 (2018).

20. Guo, Y. N., Fang, M. F. \& Zeng, K. Entropic uncertainty relation in a two-qutrit system with external magnetic field and Dzyaloshinskii-Moriya interaction under intrinsic decoherence. Quantum Inf. Process. 17, 187 (2018).

21. Li, J. Q., Bai, L. \& Liang, J. Q. Entropic uncertainty relation under multiple bosonic reservoirs with filtering operator. Quantum Inf. Process. 17, 206 (2018).

22. Ming, F. et al. Exploring uncertainty relation and its connection with coherence under the Heisenberg spin model with the Dzyaloshinskii-Moriya interaction. Quantum Inf. Process. 17, 267 (2018).

23. Zhang, Y., Zhou, Q., Fang, M., Kang, G. \& Li, X. Quantum-memory-assisted entropic uncertainty in two-qubit Heisenberg XYZ chain with Dzyaloshinskii-Moriya interactions and effects of intrinsic decoherence. Quantum Inf. Process. 17, 326 (2018).

24. Ji, Y. H., Ke, Q. \& Hu, J. J. Controlling of entropic uncertainty in open quantum system via proper placement of quantum register. Chin. Phys. B 27, 100302 (2018).

25. Yao, Y. B., Wang, D., Ming, F. \& Ye, L. Dynamics of the measurement uncertainty in an open system and its controlling. J. Phys. B At. Mol. Opt. Phys. 53, 035501 (2020).

26. Pourkarimi, M. R. Quantum correlations and entropic uncertainty relation in a three-qubit spin chain under the effect of magnetic field and DM interaction. Int. J. Quantum Inform. 16, 1850057 (2018).

27. Pourkarimi, M. R. Time evolution of quantum-memory-assisted entropic uncertainty relation and quantum correlations under dissipative environment. Int. J. Quantum Inform. 17, 1950008 (2019).

28. Huang, Z. Quantum-memory-assisted entropic uncertainty in spin models with Dzyaloshinskii-Moriya interaction. Laser Phys. Lett. 15, 025203 (2018).

29. Fang, B. L., Shi, J. \& Wu, T. Quantum-memory-assisted entropic uncertainty relation and quantum coherence in structured reservoir. Int. J. Theor. Phys. 59, 763 (2020).

30. Haddadi, S., Pourkarimi, M. R., Akhound, A. \& Ghominejad, M. Quantum correlations and quantum-memory-assisted entropic uncertainty relation in two kinds of spin squeezing models. Laser Phys. Lett. 16, 095202 (2019).

31. Pourkarimi, M. R. \& Haddadi, S. Quantum-memory-assisted entropic uncertainty, teleportation, and quantum discord under decohering environments. Laser Phys. Lett. 17, 025206 (2020).

32. Haseli, S., Dolatkhah, H., Salimi, S. \& Khorashad, A. S. Controlling the entropic uncertainty lower bound in two-qubit systems under decoherence. Laser Phys. Lett. 16, 045207 (2019).

33. Haddadi, S., Ghominejad, M., Akhound, A. \& Pourkarimi, M. R. Exploring entropic uncertainty relation and dense coding capacity in a two-qubit X-state. Laser Phys. Lett. 17, 095205 (2020).

34. Haseli, S., Haddadi, S. \& Pourkarimi, M. R. Entropic uncertainty lower bound for a two-qubit system coupled to a spin chain with Dzyaloshinskii-Moriya interaction. Opt. Quantum Electron. 52, 465 (2020).

35. Pourkarimi, M. R., Haddadi, S. \& Haseli, S. Exploration of entropic uncertainty bound in a symmetric multi-qubit system under noisy channels. Phys. Scr. 96, 015101 (2021).

36. Haseli, S., Haddadi, S. \& Pourkarimi, M. R. Probing the entropic uncertainty bound and quantum correlations in a quantum dot system. Laser Phys. 31, 055203 (2021).

37. Zidan, N. Entropic uncertainty in spin XY model with long-range interactions. Entropy 22, 837 (2020).

38. Zhang, Y., Zhou, Q., Kang, G. \& Fang, M. The dynamics of quantum-memory-assisted entropic uncertainty of two-qubit system in the XY spin chain environments with Dzyaloshinsky-Moriya interaction. Int. J. Theor. Phys. 60, 402 (2021).

39. Haddadi, S., Haseli, S., Pourkarimi, M. R. \& Mirmasoudi, F. Analyzing entropic uncertainty bound in two qubits coupled to a spin environment. Phys. Scr. 96, 075104 (2021).

40. Li, J. L. \& Qiao, C. F. The generalized uncertainty principle. Ann. Phys. (Berlin) 533, 2000335 (2021).

41. Zhang, Z. Y., Wei, D. \& Liu, J. M. Entropic uncertainty relation of a two-qutrit Heisenberg spin model in nonuniform magnetic fields and its dynamics under intrinsic decoherence. Laser Phys. Lett. 15, 065207 (2018).

42. Xiong, S. J., Sun, Z. \& Liu, J. M. Entropic uncertainty relation and quantum phase transition in spin-1/2 Heisenberg chain. Laser Phys. Lett. 17, 095203 (2020).

43. Haddadi, S., Pourkarimi, M. R. \& Haseli, S. Multipartite uncertainty relation with quantum memory. Sci. Rep. 11, 13752 (2021).

44. Haddadi, S., Ghominejad, M., Akhound, A. \& Pourkarimi, M. R. Entropic uncertainty relation and quantum coherence under Ising model with Dzyaloshinskii-Moriya interaction. Laser Phys. Lett. 18, 085204 (2021).

45. Haddadi, S., Pourkarimi, M. R. \& Wang, D. Tripartite entropic uncertainty in an open system under classical environmental noise. J. Opt. Soc. Am. B 38, 2620 (2021).

46. Haddadi, S., Pourkarimi, M. R. \& Haseli, S. Relationship between quantum coherence and uncertainty bound in an arbitrary two-qubit X-state. Opt. Quantum Electron. 53, 529 (2021).

47. Abdelghany, R. A., Mohamed, A. B. A., Tammam, M., Kuo, W. \& Eleuch, H. Tripartite entropic uncertainty relation under phase decoherence. Sci. Rep. 11, 11830 (2021).

48. Xie, Y. X. Reducing measurement uncertainty in dissipative and dephasing environments. Laser Phys. Lett. 18, 085203 (2021).

49. De Vicente, J. I. \& Sanchez-Ruiz, J. Improved bounds on entropic uncertainty relations. Phys. Rev. A 77, 042110 (2008).

50. Rudnicki, L., Walborn, S. P. \& Toscano, F. Optimal uncertainty relations for extremely coarse-grained measurements. Phys. Rev. A 85, 042115 (2012).

51. Maccone, L. \& Pati, A. K. Stronger uncertainty relations for all incompatible observables. Phys. Rev. Lett. 113, 260401 (2014).

52. Coles, P. J. \& Piani, M. Improved entropic uncertainty relations and information exclusion relations. Phys. Rev. A 89, 022112 (2014).

53. Adabi, F., Salimi, S. \& Haseli, S. Tightening the entropic uncertainty bound in the presence of quantum memory. Phys. Rev. A 93, $062123(2016)$

54. Dolatkhah, H., Haseli, S., Salimi, S. \& Khorashad, A. S. Tightening the entropic uncertainty relations for multiple measurements and applying it to quantum coherence. Quantum Inf. Process. 18, 13 (2019).

55. Xiao, Y., Jing, N., Fei, S. M. \& Li-Jost, X. Improved uncertainty relation in the presence of quantum memory. J. Phys. A Math. Theor. 49, 49LT01 (2016).

56. Chen, Z., Ma, Z., Xiao, Y. \& Fei, S. M. Improved quantum entropic uncertainty relations. Phys. Rev. A 98, 042305 (2018).

57. Li, J. L. \& Qiao, C. F. An optimal measurement strategy to beat the quantum uncertainty in correlated system. Adv. Quantum Technol. 3, 2000039 (2020).

58. Li, J. L. \& Qiao, C. F. The optimal uncertainty relation. Ann. Phys. (Berlin) 531, 1900143 (2019).

59. Ming, F. et al. Improved tripartite uncertainty relation with quantum memory. Phys. Rev. A 102, 012206 (2020). 
60. Dolatkhah, H., Haseli, S., Salimi, S. \& Khorashad, A. S. Tightening the tripartite quantum-memory-assisted entropic uncertainty relation. Phys. Rev. A 102, 052227 (2020).

61. Li, J. L. \& Qiao, C. F. Reformulating the quantum uncertainty relation. Sci. Rep. 5, 12708 (2015).

62. Chen, B. \& Fei, S. M. Sum uncertainty relations for arbitrary $N$ incompatible observables. Sci. Rep. 5, 14238 (2015).

63. Song, Q. C. \& Qiao, C. F. Stronger Schrödinger-like uncertainty relations. Phys. Lett. A 380, 2925 (2016).

64. Xiao, Y., Jing, N., Li-Jost, X. \& Fei, S. M. Weighted uncertainty relations. Sci. Rep. 6, 23201 (2016).

65. Xiao, Y. et al. Strong entropic uncertainty relations for multiple measurements. Phys. Rev. A 93, 042125 (2016).

66. Qin, H. H., Fei, S. M. \& Li-Jost, X. Multi-observable uncertainty relations in product form of variances. Sci. Rep. 6, 31192 (2016).

67. Song, Q. C., Li, J. L., Peng, G. X. \& Qiao, C. F. A stronger multi-observable uncertainty relation. Sci. Rep. 7, 44764 (2017).

68. Amico, L., Fazio, R., Osterloh, A. \& Vedral, V. Entanglement in many-body systems. Rev. Mod. Phys. 80, 517 (2008).

69. Hu, M. L. et al. Quantum coherence and geometric quantum discord. Phys. Rep. 762, 1 (2018).

70. Hu, M. L. \& Fan, H. Nonlocal advantage of quantum coherence in high-dimensional states. Phys. Rev. A 98, 022312 (2018).

71. Hu, M. L., Zhang, Y. H. \& Fan, H. Nonlocal advantage of quantum coherence in a dephasing channel with memory. Chin. Phys. B 30, 030308 (2021).

72. Bayat, A. \& Karimipour, V. Thermal effects on quantum communication through spin chains. Phys. Rev. A 71, 042330 (2005).

73. Hu, M. L., Xi, X. Q., Li, C. X. \& Qu, H. Z. Population relaxation effects on entanglement dynamics of the two-qubit spin chains. Phys. B 404, 16 (2009).

74. Hu, M. L. Disentanglement dynamics of interacting two qubits and two qutrits in an XY spin-chain environment with the Dzyaloshinsky-Moriya interaction. Phys. Lett. A 374, 3520 (2010).

75. Salberger, O. \& Korepin, V. Entangled spin chain. Rev. Math. Phys. 29, 1750031 (2017).

76. Apollaro, T. J. G., Almeida, G. M. A., Lorenzo, S., Ferraro, A. \& Paganelli, S. Spin chains for two-qubit teleportation. Phys. Rev. A 100, 052308 (2019).

77. Khedif, Y., Daoud, M. \& Sayouty, E. H. Thermal quantum correlations in a two-qubit Heisenberg XXZ spin-1/2 chain under an inhomogeneous magnetic field. Phys. Scr. 94, 125106 (2019).

78. Khedif, Y. \& Daoud, M. Pairwise nonclassical correlations for superposition of Dicke states via local quantum uncertainty and trace distance discord. Quantum Inf. Process. 18, 45 (2019).

79. Hu, M. L., Gao, Y. Y. \& Fan, H. Steered quantum coherence as a signature of quantum phase transitions in spin chains. Phys. Rev. A 101, 032305 (2020).

80. Ahadpour, S. \& Mirmasoudi, F. Thermal quantum correlations of spin chain with multiple interactions. Rev. Mex. Fis. 66, 692 (2020).

81. Khedif, Y. \& Daoud, M. Thermal quantum correlations in the two-qubit Heisenberg XYZ spin chain with Dzyaloshinskii-Moriya interaction. Mod. Phys. Lett. A 36, 2150074 (2021).

82. Khedif, Y., Errehymy, A. \& Daoud, M. On the thermal nonclassical correlations in a two-spin XYZ Heisenberg model with Dzyaloshinskii-Moriya interaction. Eur. Phys. J. Plus 136, 336 (2021).

83. Mohamed, A. B. A., Abdel-Aty, A. H. \& Eleuch, H. Dynamics of trace distance and Bures correlations in a three-qubit XY chain: Intrinsic noise model. Phys. E 128, 114529 (2021).

84. Khedif, Y., Haddadi, S., Pourkarimi, M. R. \& Daoud, M. Thermal correlations and entropic uncertainty in a two-spin system under DM and KSEA interactions. Mod. Phys. Lett. A 36, 2150209 (2021).

85. Hutton, A. \& Bose, S. Mediated entanglement and correlations in a star network of interacting spins. Phys. Rev. A 69, 042312 (2004).

86. Breuer, H. P., Burgarth, D. \& Petruccione, F. Non-Markovian dynamics in a spin star system: Exact solution and approximation techniques. Phys. Rev. B 70, 045323 (2004).

87. Krovi, H., Oreshkov, O., Ryazanov, M. \& Lidar, D. A. Non-Markovian dynamics of a qubit coupled to an Ising spin bath. Phys. Rev. A 76, 052117 (2007).

88. Ferraro, E., Breuer, H. P., Napoli, A., Jivulescu, M. A. \& Messina, A. Non-Markovian dynamics of a single electron spin coupled to a nuclear spin bath. Phys. Rev. B 78, 064309 (2008).

89. Rossini, D. et al. Bang-bang control of a qubit coupled to a quantum critical spin bath. Phys. Rev. A 77, 052112 (2008).

90. Wan-Li, Y., Hua, W., Mang, F. \& Jun-Hong, A. Tunable thermal entanglement in an effective spin-star system using coupled microcavities. Chin. Phys. B 18, 3677 (2009).

91. Arshed, N., Toor, A. H. \& Lidar, D. A. Channel capacities of an exactly solvable spin-star system. Phys. Rev. A 81, 062353 (2010).

92. Chen, Y., Shao, X. Q., Zhu, A., Yeon, K. H. \& Yu, S. C. Improving fidelity of quantum cloning via the Dzyaloshinskii-Moriya interaction in a spin network. Phys. Rev. A 81, 032338 (2010).

93. Anzà, F., Militello, B. \& Messina, A. Tripartite thermal correlations in an inhomogeneous spin-star system. J. Phys. B At. Mol. Opt. Phys. 43, 205501 (2010).

94. Militello, B. \& Messina, A. Genuine tripartite entanglement in a spin-star network at thermal equilibrium. Phys. Rev. A 83, 042305 (2011).

95. Wang, Z. H., Guo, Y. \& Zhou, D. L. Non-Markovian dynamics in a spin star system: The failure of thermalisation. Eur. Phys. J. D 67, 218 (2013).

96. Radhakrishnan, C., Lü, Z., Jing, J. \& Byrnes, T. Dynamics of quantum coherence in a spin-star system: Bipartite initial state and coherence distribution. Phys. Rev. A 100, 042333 (2019).

97. Zhao, N., Wang, Z. Y. \& Liu, R. B. Anomalous decoherence effect in a quantum bath. Phys. Rev. Lett. 106, 217205 (2011).

98. Zhao, N., Ho, S. W. \& Liu, R. B. Decoherence and dynamical decoupling control of nitrogen vacancy center electron spins in nuclear spin baths. Phys. Rev. B 85, 115303 (2012).

99. Cywiński, L., Witzel, W. M. \& Sarma, S. D. Pure quantum dephasing of a solid-state electron spin qubit in a large nuclear spin bath coupled by long-range hyperfine-mediated interactions. Phys. Rev. B 79, 245314 (2009).

100. Yang, W. \& Liu, R. B. Quantum many-body theory of qubit decoherence in a finite-size spin bath. Phys. Rev. B 78, 085315 (2008).

101. Yang, W. \& Liu, R. B. Quantum many-body theory of qubit decoherence in a finite-size spin bath. II. Ensemble dynamics. Phys. Rev. B 79, 115320 (2009).

102. Haddadi, S., Pourkarimi, M. R., Akhound, A. \& Ghominejad, M. Thermal quantum correlations in a two-dimensional spin star model. Mod. Phys. Lett. A 34, 1950175 (2019).

103. Briegel, H. J. \& Raussendorf, R. Persistent entanglement in arrays of interacting particles. Phys. Rev. Lett. 86, 910 (2001).

104. Ng, N. H. Y., Berta, M. \& Wehner, S. Min-entropy uncertainty relation for finite-size cryptography. Phys. Rev. A 86, 042315 (2012).

105. Prevedel, R., Hamel, D. R., Colbeck, R., Fisher, K. \& Resch, K. J. Experimental investigation of the uncertainty principle in the presence of quantum memory and its application to witnessing entanglement. Nat. Phys. 7, 757 (2011).

106. Cerf, N. J., Bourennane, M., Karlsson, A. \& Gisin, N. Security of quantum key distribution using $d$-level systems. Phys. Rev. Lett. 88, 127902 (2002).

107. Giovannetti, V., Lloyd, S. \& Maccone, L. Advances in quantum metrology. Nat. Photon. 5, 222 (2011).

108. Kumar, A., Haddadi, S., Pourkarimi, M. R., Behera, B. K. \& Panigrahi, P. K. Experimental realization of controlled quantum teleportation of arbitrary qubit states via cluster states. Sci. Rep. 10, 13608 (2020).

109. Uola, R., Costa, A. C. S., Chau Nguyen, H. \& Gühne, O. Quantum steering. Rev. Mod. Phys 92, 015001 (2020). 
110. Wang, D., Ming, F., Hu, M. L. \& Ye, L. Quantum-memory-assisted entropic uncertainty relations. Ann. Phys. (Berlin) 531, 1900124 (2019).

111. Türkpençe, D., Altintas, F., Paternostro, M. \& Müstecaplioğlu, Ö. E. A photonic Carnot engine powered by a spin-star network. EPL 117, 50002 (2017).

\section{Acknowledgements}

The authors acknowledge the anonymous reviewers and the editor for their helpful comments and suggestions for improving the presentation and readability of the paper.

\section{Author contributions}

S.H. has put forward the main idea and performed the calculations. S.H. and M.R.P. wrote the manuscript. S.H., M.G., A.A., and M.R.P. all contributed to the development and completion of the idea, analyzing the results, and discussions. The final draft of the manuscript was revised by S.H. and reviewed by all authors.

\section{Competing interests}

The authors declare no competing interests.

\section{Additional information}

Correspondence and requests for materials should be addressed to M.G.

Reprints and permissions information is available at www.nature.com/reprints.

Publisher's note Springer Nature remains neutral with regard to jurisdictional claims in published maps and institutional affiliations.

(c) (1) Open Access This article is licensed under a Creative Commons Attribution 4.0 International License, which permits use, sharing, adaptation, distribution and reproduction in any medium or format, as long as you give appropriate credit to the original author(s) and the source, provide a link to the Creative Commons licence, and indicate if changes were made. The images or other third party material in this article are included in the article's Creative Commons licence, unless indicated otherwise in a credit line to the material. If material is not included in the article's Creative Commons licence and your intended use is not permitted by statutory regulation or exceeds the permitted use, you will need to obtain permission directly from the copyright holder. To view a copy of this licence, visit http://creativecommons.org/licenses/by/4.0/.

(c) The Author(s) 2021 